\title{
Chemical Identification of the Radioelements Produced from Carbon and Boron by Deuteron Bombardment
}

Don M. Yost, L. N. Ridenour and K. Shinohara, Kellogg Radiation Laboratory and Gates Chemical Laboratory, California Institute of Technology

(Received December 26, 1934)

\begin{abstract}
Chemical experiments were made on the radioactive substances resulting from the bombardment of carbon and boron by deuterons. Carbon is shown to yield an isotope of nitrogen and boron an isotope of carbon. The nitrogen so formed has a half-life of 10.5 minutes while that found by Curie and Joliot on bombardment of boron with alpha-particles has a half-life of 14 minutes. These facts are discussed.
\end{abstract}

\section{INTRODUCTION}

$\mathrm{C}$ URIE and Joliot ${ }^{1}$ found that a radioactive element was produced on bombardment of boron with alpha-particles. They proposed the following nuclear reaction

$$
{ }_{5} \mathrm{~B}^{10}+{ }_{2} \mathrm{He}^{4} \rightarrow{ }_{7} \mathrm{~N}^{13}+{ }_{0} n^{1},
$$

the unstable isotope $\mathrm{N}^{13}$ disintegrating with the emission of a positron to become $\mathrm{C}^{13}$. They were able to show chemically ${ }^{2}$ that the active substance behaves like an isotope of nitrogen. The observed half-life of the activity was 14 minutes, and this has been independently confirmed by Ellis and Henderson. ${ }^{3}$ Curie and Joliot suggested that the same radioelement could be obtained by bombarding carbon with high energy deuterons.

Various investigators ${ }^{4}$ have found that the product of deuteron bombardment of carbon does exhibit radioactivity, but all are in agreement that the half-life is around 10.5 minutes. Since the nuclear reaction in this case seems very likely to be

$$
{ }_{6} \mathrm{C}^{12}+{ }_{1} \mathrm{H}^{2} \rightarrow{ }_{7} \mathrm{~N}^{13}+{ }_{0} n^{1},
$$

inasmuch as observations on the recoil nuclei indicate that a particle of about the mass of the neutron is emitted in the creation of the radioactive nucleus, ${ }^{5}$ the striking difference in halflife raises the question of the chemical nature of the element formed in this case. The three possi-

1 I. Curie and F. Joliot, Comptes rendus 198, 254 (1934).

21 . Curie and $F$. Joliot, J. de phys. et rad, $5[7], 153$ (1934).

Ellis and Henderson, Proc. Roy. Soc. A146, 206 (1934).

- Crane and Lauritsen, Phys. Rev. 45, 430 (1934); Henderson, Livingston and Lawrence, Phys. Rev. 45, 428 (1934); Hafstad and Tuve, Phys. Rev. 45, 902 (1934); Cockeroft, Int. Conf. on Physics, London, 1934.

${ }^{6}$ Cockcroft, reference 4 . bilities which exist are (a) that the radioelement formed from carbon by deuteron bombardment is not an isotope of nitrogen, but of some other element, (b) that it is a different nitrogen isotope from that formed from boron by alpha-particles, and (c) that the two radioelements are isotopic isobars; that is, both are $\mathrm{N}^{13}$, but of different structure and therefore of different disintegration probability.

While the present work was in progress, the ingenious independent experiment of Cockcroft was reported ${ }^{5}$ showing that the active gas obtained from carbon after deuteron bombardment had about the same vapor pressures as liquid nitrogen. This eliminates a number of elements, but does not eliminate $\mathrm{C}$ or $\mathrm{O}$ if present as CO.

Radioactivity has also been observed by Crane and Lauritsen ${ }^{4}$ after bombardment of boron by fast deuterons, and it seemed of interest to determine the nature of the radioelement in this case. It was known ${ }^{6}$ that while all the activity cannot be driven off a graphite target even by heating to a bright red heat, the activity produced in a target of fused boric oxide can be completely removed as a gas condensible at liquid air temperatures by heating the target to about $200^{\circ}$. The former fact is not inconsistent with the idea that the active substance is nitrogen, which is known to be strongly adsorbed on carbon; the latter is taken to indicate that the radioelement from boron is $\mathrm{CH}^{\mathrm{H}}$, formed in accordance with the reaction

$$
{ }_{5} \mathrm{~B}^{10}+{ }_{1} \mathrm{H}^{2} \rightarrow{ }_{6} \mathrm{C}^{11}+{ }_{0} n^{1} \rightarrow{ }_{6} \mathrm{~B}^{11}+{ }_{0} n^{1}+e^{+},
$$

and present in the target as $\mathrm{CO}$ or $\mathrm{CO}_{2}$.

\footnotetext{
${ }^{6}$ Crane and Lauritsen, Phys. Rev. 45, 497 (1934).
} 


\section{Experimental Method}

Carbon was bombarded in the form of graphite and boron in the form of fused boric oxide by 0.8 and $0.9 \mathrm{mev}$. deuterons accelerated in the tube developed by Crane and Lauritsen for nuclear investigations. ${ }^{7}$ Currents of about 40 microamperes were usually employed. The presence, amount, and half-life of the radioactive material were determined by quartz-fiber electroscopes constructed and kindly loaned by Professor Lauritsen, or by means of a Geiger counter whose cylindrical electrode was of aluminum about $0.2 \mathrm{~mm}$ thick.

The chemical identification was based on the well-known fact that isotopes are not appreciably separated by ordinary chemical reactions. Thus if an element consists of the fraction $\alpha$ of isotope $a$ and $(1-\alpha)$ of isotope $b$, and if the fraction $\beta$ of the total enters into a reaction, then $\beta \alpha$ and $\beta(1-\alpha)$ will be the fractions of each isotope that will have reacted. With the elements of large atomic weights this statement is almost exact, and in the case of hydrogen it is sufficiently true for the present purpose.

The experiments were always conducted on a gaseous form of the radioelement; in the case of boron this was prepared simply by heating the boric oxide target to about $600^{\circ}$ in a closed vessel and washing the resulting gases into a gasometer with air. In the case of carbon, a thin layer containing practically all the activity was scraped off the upper surface of the target and burned with about $200 \mathrm{cc}$ of air in a platinum boat in a quartz combustion tube. Air for the combustion was supplied from a specially constructed gas pipette. Small amounts of other gases could be added to the air used to burn the carbon or to the gases present in the gasometer in the experiments on boron. The following experiments were carried out; when any gas other than air was present, the fact is so stated.

\section{Carbon bombarded by deuterons}

The gases from the combustion of the carbon were passed first over heated copper oxide in all cases. They were subsequently passed through a train of solid or liquid absorbents, or they were

\footnotetext{
${ }^{7}$ Crane, Lauritsen and'Soltan, Phys. Rev. 45, 507 (1934).
}

collected over concentrated sulfuric acid in a second gas pipette similar to the first.

(1) The exit gas was passed through a train consisting of solid $\mathrm{KOH}$ (or soda lime), potash bulbs filled with 50 percent $\mathrm{KOH}, \mathrm{CaCl}_{2}$ and $\mathrm{P}_{2} \mathrm{O}_{5}$. The gas not absorbed was active. The solid $\mathrm{KOH}$ and $\mathrm{CaCl}_{2}$ showed no activity. This experiment eliminates $\mathrm{H}, \mathrm{Li}, \mathrm{Be}, \mathrm{B}$ and $\mathrm{C}$ as the active substances. The hydrogen would have been burned to $\mathrm{H}_{2} \mathrm{O}$ and this would have mixed with the water of the $\mathrm{KOH}$ solution. The small amount of water vapor from the $\mathrm{KOH}$ solution would have been absorbed by $\mathrm{CaCl}_{2}$ or $\mathrm{P}_{2} \mathrm{O}_{5}$. The oxides of $\mathrm{Li}, \mathrm{Be}$ and $\mathrm{B}$ are not volatile at room temperatures. The hydrides of boron are burned by heated air and decomposed by alkali with the formation of $\mathrm{B}_{2} \mathrm{O}_{3}$ or its salts. ${ }^{8}$ Carbon was present as $\mathrm{CO}_{2}$ and this is readily absorbed by the alkalis present in the train.

(2) The exit gases were passed through alkaline pyrogallol and then collected in the pipette. The resulting gas was active. This result eliminates oxygen as $\mathrm{O}_{2}$, since it is absorbed by the alkaline pyrogallol. If the oxygen were present as $\mathrm{NO}_{2}$, it -would undergo complete absorption in the potash.

(3) The gases resulting from the combustion of the graphite by a helium-air mixture were collected over concentrated sulfuric acid in the pipette. By passing the gas repeatedly (10 to 15 times) through alkaline pyrogallol it was freed from $\mathrm{O}_{2}$ and $\mathrm{CO}_{2}$. The gas so treated was strongly radioactive. It was next passed into an evacuated tube containing metallic calcium shavings and the calcium was then heated with a Bunsen burner. Extensive absorption of the gases took place. The tube was again evacuated to remove the helium and any other gases not absorbed by the calcium. After the contents of the tube had cooled and air had been admitted, the solid contents were removed and ground to a powder in a mortar. The powder showed strong activity. This result eliminates helium as the source of the activity, since it would be removed with the ordinary helium when the tube was evacuated.

The powder was then placed in a glass tube

B Stock, Hydrides of Boron and Silicon, Cornell Univ. Press, Ithaca, N. Y. 1933, p. 54, 63. 
equipped with a dropping funnel containing water. The gases evolved on adding water were radioactive. This result eliminates oxygen (if originally present as NO) and fluorine if present as the very unreactive $\mathrm{CF}_{4}$ or $\mathrm{C}_{2} \mathrm{~F}_{6}$. The oxygen would not be liberated as a gaseous substance in this procedure. If the fluorides of carbon were originally present, they would have been pumped out if they had not reacted with calcium, and they would have been converted to calcium fluoride and carbide had they so reacted; the calcium fluoride does not yield a volatile product in the alkaline solution such as existed on addition of water to the powder. Nitrogen would have been absorbed as $\mathrm{Ca}_{3} \mathrm{~N}_{2}$, and this substance, on treatment with water, yields ammonia.

The combined results of the chemical experiments eliminate not only all the elements of the first row of the periodic system except nitrogen as the source of the radioactivity, but also all others. On physical grounds it is possible to state that the activity must be due to one of the elements $\mathrm{H}, \mathrm{He}, \mathrm{Li}, \mathrm{Be}, \mathrm{B}, \mathrm{C}$ or $\mathrm{N}$ (unless the possibility of the emission of an electron from the carbon nucleus during bombardment is admitted, in which case $\mathrm{O}$ must be a possibility; no example of such a reaction has been established), since the total nuclear charge entering into the reaction producing the radioactive substance is 7 . The total mass entering into the reaction is probably 14 and possibly 15 (although the abundance of $\mathrm{C}^{13}$ is very small), and we may certainly presume from the experiments on recoil radioactive nuclei ${ }^{5}$ that the mass number of the radioactive product is surely not larger than 14 and is probably 13 . Inasmuch as the only particles emitted from carbon under deuteron bombardment in observable numbers are known to be neutrons and protons, the radioactivity is probably due to $\mathrm{C}$ or $\mathrm{N}$. Since we may assume in analogy with all cases of artificial radioactivity that the active substance is an isotope of mass number different from those known by mass spectra, it is likely to be $\mathrm{C}^{14}$ or $\mathrm{N}^{13}$, and the latter is the more probable on the ground of the rarity of $\mathrm{C}^{13}$, as well as from the fact that it emits positrons. The present experiment seems definitely to have established that it is nitrogen, and we may then infer that it is likely to be $\mathrm{N}^{13}$.
Measurements on the half-life of the active gas at various stages in the chemical treatment yielded concordant values around 10.5 minutes, indicating that the activity is due to a single substance.

\section{Boron bombarded by deuterons}

The total mass entering into this reaction may be 12 or 13 , corresponding to $\mathrm{B}^{10}$ or $\mathrm{B}^{11}$. The total charge is 6 , and we may again for safety admit the possibility of the emission of a negative electron from the nucleus. On physical grounds, the activity may, then, be due to $\mathrm{H}$, $\mathrm{He}, \mathrm{Li}, \mathrm{Be}, \mathrm{B}, \mathrm{C}$ or $\mathrm{N}$. Since a heavy particle must be emitted in the creation of the radioelement, ${ }^{5}$ the total mass of the product must be 12 or smaller. The chemical experiments were as follows:

(1) A small amount of carbon dioxide was added to a sample of gas collected in the gasometer. One-half of the gas was tested and found to be active. The other half was passed repeatedly through $\mathrm{KOH}$ solution, and the resulting gas showed an activity of about one-third that of the first half, after correcting for known decay which took place while the operation was carried out. Some of the active materials evidently had been absorbed by the potash. Since $\mathrm{Li}$ and $\mathrm{Be}$ compounds are involatile these elements are eliminated as possible sources of all the activity.

(2) To a $150 \mathrm{cc}$ sample of gas in the gasometer about $10 \mathrm{cc}$ of carbon monoxide were added, and the mixture was then passed over heated cupric oxide. Half of the resulting gas was tested with an electroscope and found to be active. The other half was passed several times through 50 percent $\mathrm{KOH}$ solution and then into the electroscope. The resulting gas was inactive. These two results eliminate $\mathrm{B}, \mathrm{N}_{2}$ and $\mathrm{O}_{2}$ as possibilities, since the volatile hydrides of $\mathbf{B}$ would burn to $\mathrm{B}_{2} \mathrm{O}_{3}$ on the hot $\mathrm{CuO}$ or be decomposed by the alkali, ${ }^{8}$ and $\mathrm{N}_{2}$ and $\mathrm{O}_{2}$ would not be absorbed by $\mathrm{KOH}$.

(3) Experiment 2 was repeated after $10 \mathrm{cc}$ of helium had been added to the gas sample in the gasometer. The activity was absorbed by $\mathrm{KOH}$. This result excludes $\mathrm{He}$ as the source of the activity since it is not absorbed by alkalies.

(4) A sample of gas to which $\mathrm{CO}$ and $\mathrm{NO}_{2}$ had been added was passed over hot $\mathrm{CuO}$ and through a strong solution of $\mathrm{KMnO}_{4}$ in $3 \mathrm{~N}$ sulfuric acid. 
The resulting gas was active. The experiment was repeated but without passing over hot $\mathrm{CuO}$. The result was the same. These results excluded $\mathrm{H}_{2}$, and $\mathrm{N}$ as $\mathrm{NO}$ or $\mathrm{NO}_{2}$, for hydrogen would burn to water and be mixed with the water in the permanganate solution; $\mathrm{NO}$ or $\mathrm{NO}_{2}$ would be oxidized completely to $\mathrm{HNO}_{3}$ by the acidified permanganate. ${ }^{2}$ Fluorine, if present as $\mathrm{BF}_{3}$, would be completely hydrolyzed and absorbed by the aqueous solution. Boron, if present as volatile hydrides, would be oxidized and decomposed by the heated $\mathrm{CuO}$ or hydrolyzed by the solution.

The results of the chemical experiments eliminate all elements in the first row of the periodic system except carbon. The gas driven from

' Klemenc and Bunzl, Zeits. f. anorg. Chemie 122, 315 (1922). the boric oxide target has the properties of a mixture of carbon dioxide and carbon monoxide. Just why the newly formed carbon atoms should be oxidized partly to monoxide and partly to dioxide is not evident. It was not determined whether the two compounds were always formed in the same proportions.

Measurements on the half-life of the radioactive gas both before and after chemical treatment yielded the value 20.5 minutes in all cases, indicating that the activity is due to a single substance. Since $\mathrm{C}^{12}$ is stable, on the physical grounds outlined above, this substance is probably $\mathrm{C}^{\mathrm{H}}$.

We wish to express here our thanks to the Seeley W. Mudd fund for the financial support which made this work possible.

\title{
Energy Levels of a Symmetrical Double Minima Problem with Applications to the $\mathrm{NH}_{3}$ and $\mathrm{ND}_{3}$ Molecules ${ }^{1}$
}

\author{
Millard F. Manning, Department of Physics, Massachusetis Institute of Technology
}

(Received January 14, 1935)

\begin{abstract}
The energy levels for a potential energy $V=-C \operatorname{sech}^{2} r / 2 \rho+D \operatorname{sech}^{4} r / 2 \rho$ have been obtained by numerical solution of continued fractions. Values of the constants have been found such that the computed energy levels agree satisfactorily with the experimental values for the $\mathrm{NH}_{3}$ and $\mathrm{ND}_{3}$ molecules. The height of the ammonia pyramid is found to be $0.37 \mathrm{~A}$, the height of the potential hill is found to be $2076 \mathrm{~cm}^{-1}$, and the dissociation energy is about five volts.
\end{abstract}

$I^{T}$

$\mathrm{T}$ has been found possible to obtain a satisfactory interpretation of the infrared spectrum of ammonia by assuming that the molecule is shaped like a symmetrical pyramid. The potential energy as a function of the distance of the nitrogen atom from the plane of the hydrogens has the general shape indicated in Fig. 1. Whenever there are symmetrical double minima in the potential energy, the energy levels below the top of the center of the hill will be double, and above the top of the hill the levels for which

\footnotetext{
${ }^{1}$ A preliminary report of these results is given in an abstract, Phys. Rev. 46, 335 (1934).
}

the wave functions are symmetric will be shifted upward with respect to the levels for which the wave functions are antisymmetric.

Quantitative calculations of the behavior of the energy levels of a symmetrical double minima problem have been carried out by several authors. ${ }^{2}$ The assumed forms of potential energy have been somewhat artificial, and except for the paper of Morse and Stueckelberg the methods used have been approximate. For these

2 Morse and Stueckelberg, Helv. Phys. Acta 4, 337 (1931); Dennison and Uhlenbeck, Phys. Rev. 41, 313 (1932); Rosen and Morse, Phys. Rev. 42, 210 (1932). 